\title{
Electrical Conduction Properties of SiC Modified by Femtosecond Laser
}

\author{
Takuto Ito ${ }^{1}$, Manato Deki ${ }^{1}$, Takuro Tomita ${ }^{*}{ }^{1}$, Shigeki Matsuo ${ }^{1}$, Shuichi Hashimoto ${ }^{1}$, \\ Takahiro Kitada ${ }^{2}$, Toshiro Isu ${ }^{2}$, Shinobu Onoda ${ }^{3}$ and Takeshi Ohshima ${ }^{3}$ \\ ${ }^{1}$ Department of Ecosystem Engineering, The University of Tokushima, \\ Tokushima, 770-8506, Japan \\ E-mail: tomita@eco.tokushima-u.ac.jp \\ ${ }^{2}$ Center of Frontier Research of Engineering, The University of Tokushima, \\ Tokushima 770-8506, Japan \\ ${ }^{3}$ Japan Atomic Energy Agency, Takasaki 370-1292, Japan
}

\begin{abstract}
We have observed the electrical conduction properties of silicon carbide $(\mathrm{SiC})$ that was locally modified by femtosecond laser. The current-voltage $(I-V)$ characteristics of laser-modified regions were measured. Intriguingly, when the polarization of the laser beam was parallel to the scanning direction, the resistance of the modified region decreased with increasing the irradiated fluence. The resistance of the region irradiated at a fluence of $86 \mathrm{~J} / \mathrm{cm}^{2}$ decreased by more than six orders of magnitude compared with the non-irradiated one. In contrast, when the polarization of the laser beam was perpendicular to the scanning direction, the resistance of the modified region did not show the significant reduction. From the scanning electron microscope observations and Raman spectroscopy, we suppose that the difference of the resistance for each polarization direction is due to the difference of the chemical composition generated in laser modified region.

DOI:10.2961/jlmn.2012.01.0003
\end{abstract}

Keywords: Silicon Carbide, Femtosecond Laser, Electrical Conduction Properties, Ripple, Raman Spectroscopy.

\section{Introduction}

In recent years, silicon carbide $(\mathrm{SiC})$, which is a wideband-gap semiconductor and transparent to visible light, attracts much attention as the next-generation semiconductor material. On the other hand, femtosecond laser processing is possible to process inside transparent materials, such as silica, polyimide and wide-band-gap semiconductors without destroying the surface of those materials [1]. We have been trying to control the local electric conductivities of $\mathrm{SiC}$ by femtosecond laser. If we can intentionally control the local electric conductivities in $\mathrm{SiC}$, the combination of femtosecond laser modification technique and transparent properties of $\mathrm{SiC}$ leads to the three-dimensional electric circuit fabrication method in $\mathrm{SiC}$.

We have been reported that the femtosecond laser modification enhances the local electric conductivities in $\mathrm{SiC}$ [2]. However, we have not discussed the effect of irradiation polarization in the conductivity. In this paper, we report the electric conduction properties of $\mathrm{SiC}$ modified by femtosecond laser. In particular, we discuss the polarization dependence of the resistance in modified region and the effect of ion-implanted metal contact on the measured resistivity for the development of laser modification techniques in $\mathrm{SiC}$.

\section{Experiments}

We used a semi-insulating single-crystalline $6 \mathrm{H}-\mathrm{SiC}$ (SiXON) wafer (Si face) locally modified by femtosecond laser irradiation. For electrode fabrication, phosphorus ions $\left(\mathrm{P}^{+}\right)$at energies of 60,90 and $140 \mathrm{keV}$ were locally implanted in the samples (a mean concentration of $\mathrm{P}$ : $5 \times 10^{19} / \mathrm{cm}^{3}$ ), and subsequently, aluminum (Al) was deposited on the P-ion-implanted regions. The distance between the adjacent $\mathrm{Al}$ contacts was $1.02 \mathrm{~mm}$. The specific resistance of these samples before laser irradiation was higher than $10^{5} \Omega \mathrm{cm}$. For the laser irradiation experiments, the light source used to modify samples surface was a $1 \mathrm{kHz}$ Ti: sapphire regenerative amplifier based on chirped pulse amplification (Spectra Physics, Spitfire). The wavelength, pulse duration and repetition rate were $800 \mathrm{~nm}, 130 \mathrm{fs}$ and $1 \mathrm{kHz}$, respectively. The laser beam was focused on the samples surface by using a 10x objective lens with the numerical aperture (N.A.) of 0.40 through optical microscope (Olympus IX-70). The femtosecond laser was irradiated in a straight line between the Al contacts by using a motorized mechanical stage (Suruga Seiki, KS701-20LHD) with a scanning speed of $100 \mu \mathrm{m} / \mathrm{s}$. The radius of the laser beam on the samples surface was approximately $3.0 \mu \mathrm{m}$ evaluated by the $\mathrm{D}^{2}$ method [3]. Thus, the accumulated number of irradiated pulses for one site was approximately 60 shots. The pulse fluence was varied from 1.0 to $86 \mathrm{~J} / \mathrm{cm}^{2}$. The polarization (E, electric field) of the laser beam was adjusted to be parallel to the scanning direction (S) (S // E) or perpendicular to the scanning direction $(\mathrm{S} \perp \mathrm{E})$ by using a half wavelength plate. The current-voltage (I-V) characteristics and as a result the resistance of the lasermodified region between the $\mathrm{Al}$ contacts were measured by a semiconductor parameter analyzer (Hewlett Packard, $4156 \mathrm{~A})$ with the applied voltage from -0.1 to $+0.1 \mathrm{~V}$. The debris which were generated by femtosecond laser irradiation around irradiated regions were removed by ultrasonic cleaning in acetone, methanol, and distilled 

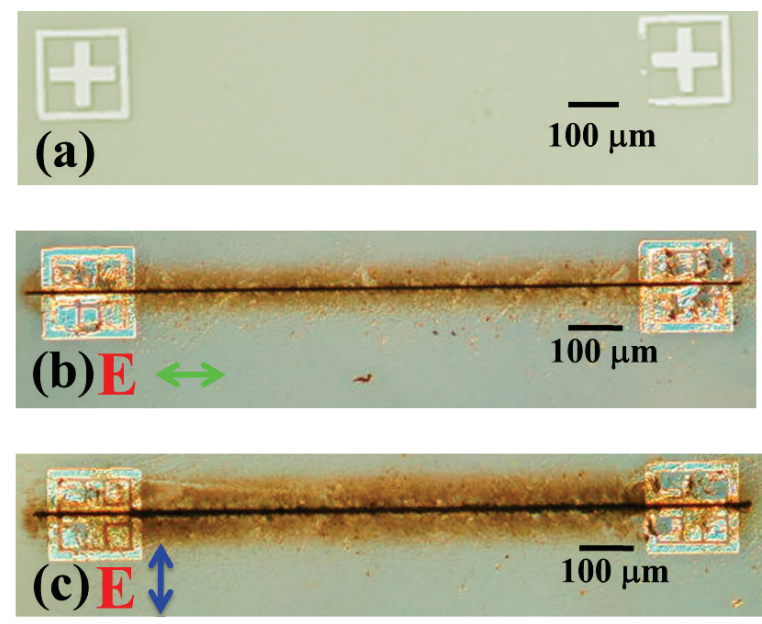

Figure 1 The optical micrograph of samples. The irradiation condition were (a) non-irradiated, (b) $14 \mathrm{~J} / \mathrm{cm}^{2}$ (S //E), (c) 14 $\mathrm{J} / \mathrm{cm}^{2}(\mathrm{~S} \perp \mathrm{E})$.

water. The sample surface was observed by using a scanning electron microscope (SEM, Hitachi, S-4700) and a confocal Raman microscope (Renishaw, InVia Raman Microscopy) for the morphological observation and the chemical composition analysis of the laser-modified region. The light source used to measure the Raman spectra was diode pumped solid state (DPSS) laser with the wavelength of $532 \mathrm{~nm}$.

\section{Results and discussion}

\subsection{Current-voltage characteristics of the modified region}

Figure 1 shows an optical micrograph of sample. Figure 1 (a) shows the surface of the non-irradiated one. Figure 1 (b) and (c) show the laser-modified sample with the irradiated fluence of $14 \mathrm{~J} / \mathrm{cm}^{2}$ for $\mathrm{S} / / \mathrm{E}((\mathrm{b}))$ and $\mathrm{S} \perp \mathrm{E}$ ((c)). From the optical observation, it was confirmed that sample surface was modified by femtosecond laser without the significant difference in the appearance between $\mathrm{S} / / \mathrm{E}$ and $\mathrm{S} \perp \mathrm{E}$ condition.

Figure 2 shows the $I-V$ characteristics of femtosecond laser irradiated region in $\mathrm{SiC}$. The laser polarization was $\mathrm{S}$ //E. In Fig. 2, the irradiated fluence used to modify the sample were $1.5 \mathrm{~J} / \mathrm{cm}^{2}$ [red], $6.7 \mathrm{~J} / \mathrm{cm}^{2}$ [green], and $33 \mathrm{~J}$ $/ \mathrm{cm}^{2}$ [blue]. The $I-V$ characteristics of the sample irradiated with the fluence of $1.5 \mathrm{~J} / \mathrm{cm}^{2}$ was similar to that of the nonirradiated one. With increasing laser power, the current

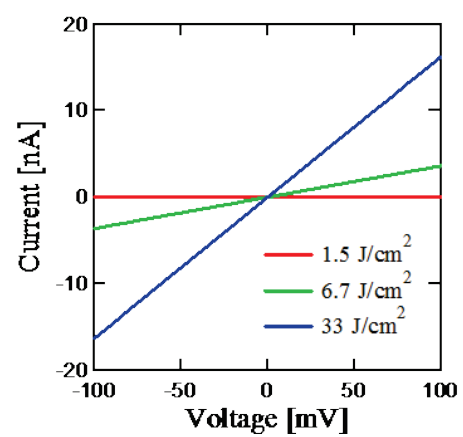

Figure 2 The $I-V$ characteristics of femtosecond laser irradiated region in $\mathrm{SiC}(\mathrm{S} / / \mathrm{E})$. increases. And, for the irradiated fluence of $33 \mathrm{~J} / \mathrm{cm}^{2}$, the current increased up to more than $10 \mathrm{nA}$ with an applied voltage of $+0.1 \mathrm{~V}$. When the sample surface was irradiated with femtosecond laser, the debris was generated around irradiated region. To discuss the effect of the debris on the electrical conductivity, the debris was removed by the cleaning processes as mentioned previously. The $I-V$ characteristics did not change between before and after cleaning. This result indicates that debris does not affect the electrical conductivity. We will focus on the resistance as the indicator of the electrical conductivity in the following paragraphs.

\subsection{Irradiation fluence dependence of the resistance}

Figure 3 shows a log-log plot of the irradiated fluence and the resistance for each laser polarization in femtosecond laser irradiated region. The green dots and blue dots indicate $\mathrm{S} / / \mathrm{E}$ and $\mathrm{S} \perp \mathrm{E}$, respectively. The resistance between the $\mathrm{Al}$ contacts before laser irradiation is higher than $10^{11} \Omega$. For the irradiated fluence lower than $5 \mathrm{~J} / \mathrm{cm}^{2}$, the significant difference of the resistance was not observed for both laser polarizations. In the irradiated fluence lower than $2.0 \mathrm{~J} / \mathrm{cm}^{2}$, the resistance was similar to that of the non-irradiated one. The resistance of $\mathrm{S} / / \mathrm{E}$ condition decreased with increasing the irradiated fluence. The resistance of $\mathrm{S} / / \mathrm{E}$ condition at the irradiated fluence of $86 \mathrm{~J} / \mathrm{cm}^{2}$ decreased by more than six orders of magnitude compared with the non-irradiated one. In contrast, for $\mathrm{S} \perp \mathrm{E}$ condition, the resistance did not decrease as in the case of $\mathrm{S} / / \mathrm{E}$ even for the higher fluence. For the irradiated fluence higher than $20 \mathrm{~J} / \mathrm{cm}^{2}$, the contrast of the resistance between $\mathrm{S} / / \mathrm{E}$ and $\mathrm{S} \perp \mathrm{E}$ conditions was more than five orders of magnitude.

The decrease of the resistance in $\mathrm{S} / / \mathrm{E}$ condition is supposed to be caused by a material phase transition induced by laser irradiation [2]. The formation of spontaneously formed periodic structures, so-called ripples, by femtosecond laser irradiation on single-crystal $\mathrm{SiC}$ was studied by SEM and transmission electron microscopy (TEM) [4]. Such ripples are often found on various materials after laser irradiation [5-11]. It was reported that coarse ripples are formed for a higher fluence compared with fine ripples, and the period of coarse ripple is

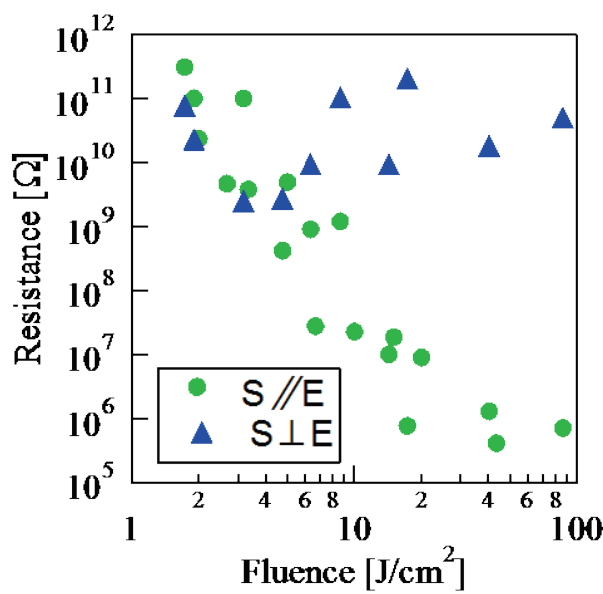

Figure 3 The irradiated fluence dependence of the resistance of the modified regions for each polarization configurations. 
determined from the surface plasmon resonance and the subsequent grating assisted effect $[6-8,12]$.

\subsection{SEM observation of the modified region}

Figure 4 shows the SEM images of femtosecond lasermodified sample surfaces irradiated with the pulse fluence of $2.5 \mathrm{~J} / \mathrm{cm}^{2}$ ((a), (b)) and $34 \mathrm{~J} / \mathrm{cm}^{2}$ ((c), (d)). The polarizations of the laser beam of Fig. 4 ((a), (c)) and ((b), (d)) were $\mathrm{S} / / \mathrm{E}$ and $\mathrm{S} \perp \mathrm{E}$, respectively. In Fig. 4 (a), (b), the fine ripple structure was formed on the whole area of the laser-modified surface. It was found that the fine ripple structure was formed on the entire area of the lasermodified surface with the fluence lower than about $5 \mathrm{~J} / \mathrm{cm}^{2}$ for both laser polarizations. The direction of the fine ripple structure was perpendicular to polarization for both laser polarizations. In Fig. 4 (c), the dot-like structure was observed on the periphery of the laser-modified surface and the deep grooves were observed on the central region of the laser modified surface. It was found that the dot-like structure was observed on the periphery of the lasermodified surface and the deep grooves on the central region of the laser-modified surface for the fluence higher than $8.0 \mathrm{~J} / \mathrm{cm}^{2}$ in $\mathrm{S} / / \mathrm{E}$ condition. In the periphery of the laser modified surface, small amount of the debris which remained after the cleaning was observed. In Fig. 4 (d), the fine ripple structure was still formed on almost the whole area of the laser-modified surface. For the any irradiated fluence for $\mathrm{S} \perp \mathrm{E}$ condition, only the fine ripple structure was observed. The deep grooves, which are formed for the fluence higher than $8.0 \mathrm{~J} / \mathrm{cm}^{2}$ in $\mathrm{S} / / \mathrm{E}$ condition, was not observed in $\mathrm{S} \perp \mathrm{E}$ condition. The direction of the fine ripple structure formed in Fig. 4 (d) is the same as the direction of (b). The resistance is lower for $2.5 \mathrm{~J} / \mathrm{cm}^{2}$ (Fig. 4 (a)) and higher for $34 \mathrm{~J} / \mathrm{cm}^{2}$ (Fig. 4 (c)), indicating that the central area of the laser-modified surface for $34 \mathrm{~J} / \mathrm{cm}^{2}$ (deep grooves, Fig. 4 (c)) contributes to the high electric conductivity. From this fact, it is supposed that the resistance is high when the fine ripple structure was formed in the entire region. The coarse ripple was not observed for any irradiated fluence in $\mathrm{S} \perp \mathrm{E}$ condition. One possible reason why the coarse ripple was not observed for all the irradiation condition is that high NA of the objective lens causes large distribution of the incident angle. On the other hand, it has reported that the formation of the fine ripple

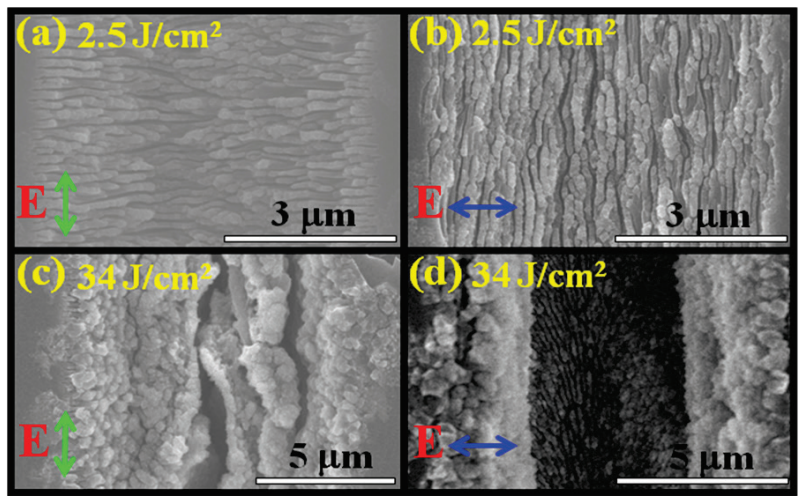

Figure 4 SEM images of sample surface of the femtosecond laser irradiated $\mathrm{SiC}$. (a) $2.5 \mathrm{~J} / \mathrm{cm}^{2}$ (S //E), (b) $2.5 \mathrm{~J} / \mathrm{cm}^{2}(\mathrm{~S} \perp \mathrm{E})$, (c) $34 \mathrm{~J} / \mathrm{cm}^{2}(\mathrm{~S} / / \mathrm{E})$ and (d) $34 \mathrm{~J} / \mathrm{cm}^{2}(\mathrm{~S} \perp \mathrm{E})$. does not significantly depend on the incident angle [11]. Thus, it is supposed that fine ripple is formed on the sample surface even for the objective lens with a higher N.A.

\subsection{Raman spectroscopic study of the modified region}

We suppose that the similar type of phase transition that was associated with the formation of the coarse ripple may occur in the central area of Fig. 4 (c), while the surface corrugation was destroyed due to the high N.A. We have reported that ripples are composed of amorphous $\mathrm{SiC}$, amorphous silicon, and amorphous carbon [13]. The ratio of the amorphous silicon to the amorphous $\mathrm{SiC}$ is abruptly increased in the coarse ripple compared with the fine ripple. It is supposed that the decrease in the resistance down to $10^{5} \Omega$ may be caused by the generation of amorphous silicon and/or carbon with increasing the irradiated fluence. To confirm this point, we investigated the chemical composition of laser-modified region by Raman spectroscopy. Figure 5 shows the Raman spectra of lasermodified region for each irradiation condition. Figure 5 (a) shows Raman spectra of non-irradiation [red], $\mathrm{S} \perp \mathrm{E}$ [blue], and $\mathrm{S} / / \mathrm{E}$ [green] at the fluence of $1.7 \mathrm{~J} / \mathrm{cm}^{2}$. Figure $5(\mathrm{~b})$ shows the Raman spectra of $\mathrm{S} / / \mathrm{E}$ [green] and $\mathrm{S} \perp \mathrm{E}$ [blue] at the irradiated fluence of $8.6 \mathrm{~J} / \mathrm{cm}^{2}$. The Raman spectra of both laser polarizations in Fig. 5 (a) were similar to that of non-irradiated one. The chemical composition of lasermodified region for both laser polarizations at the irradiated fluence of $1.7 \mathrm{~J} / \mathrm{cm}^{2}$ was single crystal $\mathrm{SiC}$ (c-SiC). In Fig. 5 (b), the significant difference of Raman spectra was observed for each laser polarization. The Raman spectra of $\mathrm{S} \perp \mathrm{E}$ condition with the irradiated fluence of $8.6 \mathrm{~J} / \mathrm{cm}^{2}$ was similar to that of non-irradiated one. The chemical composition of laser-modified region for $\mathrm{S} \perp \mathrm{E}$ condition
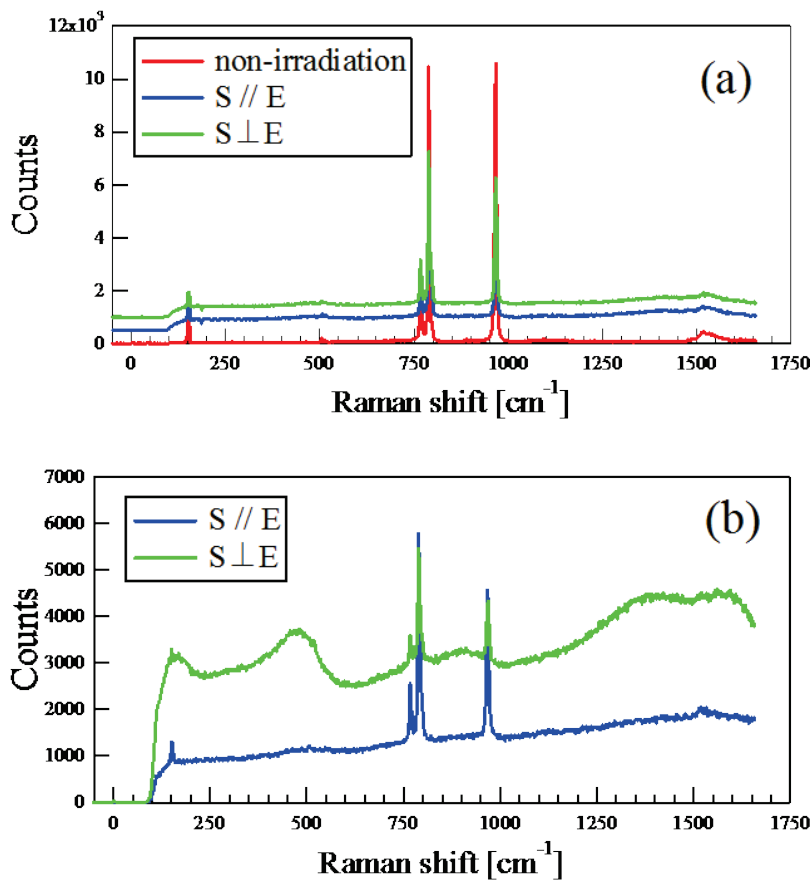

Figure 5 Raman spectra of laser-modified region for each polarization condition. (a) non-irradiated [red], S //E [green], $\mathrm{S} \perp \mathrm{E}$ [blue] at the fluence of $1.7 \mathrm{~J} / \mathrm{cm}^{2}$ (b) $\mathrm{S} / / \mathrm{E}$ [green], $\mathrm{S} \perp \mathrm{E}$ [blue] at the fluence of $8.6 \mathrm{~J} / \mathrm{cm}^{2}$. The spectra are shifted along the ordinate for the convenient display. 
with the irradiated fluence of $8.6 \mathrm{~J} / \mathrm{cm}^{2}$ remained c-SiC. In contrast, the Raman spectra of $\mathrm{S} / / \mathrm{E}$ condition with the irradiated fluence of $8.6 \mathrm{~J} / \mathrm{cm}^{2}$ showed some broad peaks. The $260 \mathrm{~cm}^{-1}$ and $480 \mathrm{~cm}^{-1}$ peaks can be attributed to amorphous silicon (a-Si), and the $770 \mathrm{~cm}^{-1}$ and $880 \mathrm{~cm}^{-1}$ peaks to amorphous $\mathrm{SiC}$ (a-SiC) [13]. The bands correspond to amorphous graphite also appeared around $1350 \mathrm{~cm}^{-1}$ and $1590 \mathrm{~cm}^{-1}$. This means that the modified region for $\mathrm{S} / / \mathrm{E}$ condition with the fluence of $8.6 \mathrm{~J} / \mathrm{cm}^{2}$ was composed of a-SiC, a-Si and a-C. From these facts, it is concluded the generation of a-SiC, a-Si and a- $\mathrm{C}$ causes the drastic decrease of electric conductivity.

In $\mathrm{S} / / \mathrm{E}$ condition, the structure of laser irradiated region changes from fine ripple structure to deep grooves with increasing the irradiated fluence. However, in $\mathrm{S} \perp \mathrm{E}$ condition, the structure of laser-modified region still remains fine ripple structure even if the irradiated fluence increases. From the Raman spectra, it was found that the chemical composition of laser modified region in $\mathrm{S} / / \mathrm{E}$ condition changes from c-SiC to a-SiC, a-Si and a-C as the irradiated fluence increases. However, in $\mathrm{S} \perp \mathrm{E}$ condition, the chemical composition of laser-modified region still remained in $\mathrm{c}-\mathrm{SiC}$ even if the irradiated fluence increases. Although the reason why the composition is different between these polarization configurations is not clear, we tentatively suppose that the transient depth profile of the modified spot may affect the difference of optical absorption between these polarization configurations.

\subsection{The effect of contact resistance}

The resistance plotted in Fig. 3 includes the resistance of the $\mathrm{Al}$ contacts on the both side of the modified regions. It is important to evaluate the contact resistance in order to precisely evaluate the resistance of the modified region. We measured the distance dependence of the resistance to evaluate the contact resistance. Three distances between $\mathrm{Al}$ contacts of $1.02 \mathrm{~mm}, 2.22 \mathrm{~mm}$ and $3.42 \mathrm{~mm}$ were employed to evaluate the distance dependence. Experimental conditions other than the distance between $\mathrm{Al}$ contacts were the same. Figure 6 shows the relationship between the distance and the resistance of laser-modified region for each the irradiated fluence. The results in Fig.6 showed a linear relationship. Here, the values of the intercept correspond to the contacts resistivity for each irradiation condition. The value of the intercept for each the irradiated fluence was from about $2.4 \times 10^{4}$ to about $8.6 \times 10^{4}$

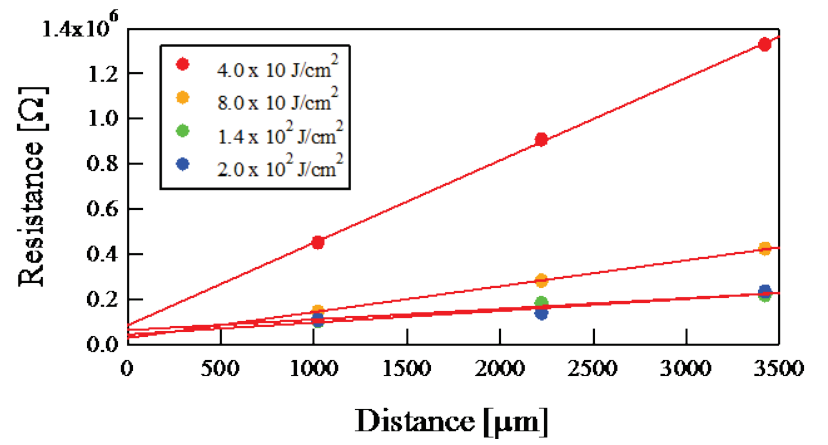

Figure 6 The relationship between the distance and the resistance of laser modified region for the irradiated fluence of $4.0 \times 10 \mathrm{~J} / \mathrm{cm}^{2}$ (red), $8.0 \times 10 \mathrm{~J} / \mathrm{cm}^{2}$ (yellow), $1.4 \times 10^{2} \mathrm{~J}$ $/ \mathrm{cm}^{2}$ (green) and $2.0 \times 10^{2} \mathrm{~J} / \mathrm{cm}^{2}$ (blue).
$\Omega$. The variation of these values is supposed to be reasonably constant for each condition. Thus, by averaging these values, the contact resistance is estimated to be about $5 \times 10^{4} \Omega$. This value is far lower than the observed resistance in Fig. 3. Thus, we conclude that the contact resistance does not significantly affect on the measured resistance of the modified regions in Fig. 3.

\section{Conclusion}

The $I-V$ characteristics of the femtosecond laser modified region in $\mathrm{SiC}$ were systematically investigated. To evaluate the change in electrical conductivities in semiinsulating $\mathrm{SiC}, \mathrm{Al}$ contacts were deposited on the phosphorus $\left(\mathrm{P}^{+}\right)$ion-implanted region of $\mathrm{SiC}$. The sample surface was modified by femtosecond laser irradiation with different the polarization configurations ( $\mathrm{S} / / \mathrm{E}$ and $\mathrm{S} \perp \mathrm{E})$. The resistance of laser-modified region for $\mathrm{S} / / \mathrm{E}$ condition decreased with increasing the irradiated fluence. The resistance at the irradiated fluence of $86 \mathrm{~J} / \mathrm{cm}^{2}$ for $\mathrm{S} / / \mathrm{E}$ condition decreased by more than six orders of magnitude compared with the non-irradiated one. In contrast, the resistance of laser-modified region for $\mathrm{S} \perp \mathrm{E}$ condition did not decrease as in the case of $\mathrm{S} / / \mathrm{E}$ even if the irradiated fluence increases. We suppose that the difference of this fluence dependence on the resistivity is caused by the difference of the chemical composition generated in laser modified region. The generation of a-SiC, a-Si and a-C by femtosecond laser irradiation especially induces the drastic reduction of the conductivity.

\section{Acknowledgments}

We acknowledge Mr. Naoya Iwamoto at JAEA (and also The University of Electro-Communications) for helpful assistance regarding $\mathrm{P}^{+}$implantation and $\mathrm{Al}$ evaporation. This research is partially supported by a Grant-in-Aid for Scientific Research from the Ministry of Education, Culture, Sports, Science and Technology (Grant No. 19760035) and the MURATA science foundation.

\section{References}

[1] S. Matsuo, S. Kiyama, Y. Shichijo, T. Tomita, S. Hashimoto, Y. Hosokawa and H. Masuhara: Appl. Phys. Lett., 93, (2008) 051107.

[2] M. Deki, T. Ito, M. Yamamoto, T. Tomita, S. Matsuo, S. Hashimoto, T. Kitada, T. Isu, S. Onoda and T. Ohshima: Appl. Phys. Lett., 98, (2011) 133104.

[3] J. M. Liu: Opt. Lett., 7, (1982) 196.

[4] T. Tomita, K. Kinoshita, S. Matsuo and S. Hashimoto: Jpn. J. Appl. Phys., 45, (2006) L444.

[5] M. Birnbaum: J. Appl. Phys., 36, (1965) 3688.

[6] J. E. Sipe, J. F. Young, J. S. Preston and H. M. Driel: Phys. Rev. B 27, 1141 (1983).

[7] J. F. Young, J. S. Preston, H. M. Driel, and J. E. Sipe, Phys. Rev. B, 27, (1983) 1155.

[8] J. F. Young, J. E. Sipe and H. M. Driel: Phys. Rev. B, 30, (1984) 2001.

[9] A. M. Ozkan, A. P. Malshe, T. A. Railkar, W. D. Brown, M. D. Shirk and P. A. Molian: Appl. Phys. Lett., 75, (1999) 3716.

[10] A. Borowiec and H. K. Haugen: Appl. Phys. Lett., 82, (2003) 4462 
[11] W. Kautek, P. Rudolph, G. Daminelli and J. Krüger: Appl. Phys. A: Mater. Sci. Process., 81, (2005) 65.

[12] M. Huang, F. L. Zhao, Y. Cheng, N. Xu, and Z. Xu: ACS Nano, 3, (2009) 4062.
[13] M. Yamaguchi, S. Ueno, R. Kumai, K. Kinoshita, T. Murai, T. Tomita, S. Matsuo and S. Hashimoto: Appl. Phys. A: Mater. Sci. Process., 99, (2010) 23.

(Received: June 7, 2011, Accepted: December 12, 2011) 